\title{
Corrosion detection by multi-step genetic algorithm
}

\author{
K. Amaya ${ }^{1}$, M. Ridha ${ }^{1} \&$ S. Aoki ${ }^{2}$ \\ ${ }^{1}$ Tokyo Institute of Technology, Ookayama, Meguro, Tokyo, Japan \\ ${ }^{2}$ Toyo University, Kujirai, Kawagoe, Saitama, Japan
}

\begin{abstract}
A multi-step genetic algorithm is developed to eliminate the limitations of the inverse analysis method for detecting the corrosion profile on the steel matrices from a small number of potential data on the concrete structure. The corrosion profile represents the the number, locations and shapes of plural corrosion parts on the steel matrices. It was modeled into a binary string which is defined by discretizing the steel matrices into a suitable number of segments using a certain resolution. Each segment is encoded by one binary bit. A tree structure is employed in the multi-step genetic algorithm and the examination is localized for root and each branch. By this approach, we can avoid a long binary string that is required to encode the segments when a large examination area is discretized using a required resolution. Hence, for each step of examination, the standard genetic algorithm can be locally applied and performed efficiently. We demonstrate the effectiveness of the proposed method using an example of numerical simulation.
\end{abstract}

\section{Introduction}

Detection of reinforcement corrosion in the early stage is important to reduce maintenance cost and increase the durability of the concrete structure [1].

The boundary element inverse analysis [2] has been introduced to estimate the locations and sizes of plural corrosion in the concrete structure by modeling them into a set of unknown parameters. The inverse analysis was carried out by minimizing the cost-function using a down-hill simplex method. However, a such method required to predetermine the number and shapes of plural corroded parts. In addition, some difficulties were occured related with an appropriate initial guess of the set of unknown parameters. 


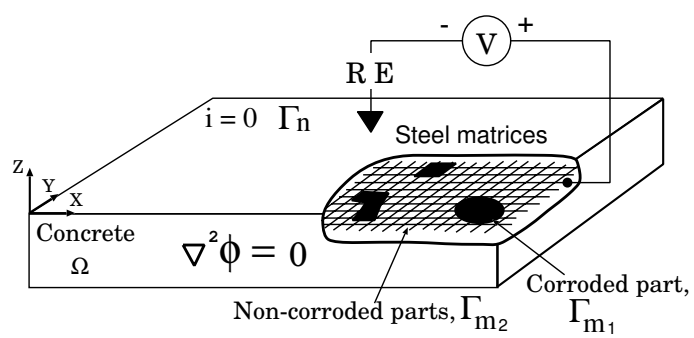

Figure 1: Model of concrete domain for corrosion analysis.

The purpose of this research is to develop a multi-step genetic algorithm (GA) for eliminating the above limitations. In this method, A tree structure is employed and the examination was carried out separately for the root and the branches. For each step of examination, the corrosion profile is modeled into a binary string which is defined by discretizing the steel matrices of the examination area into a suitable number of segment using a certain resolution.

\section{Numerical analysis for corrosion detection}

Let us consider that the concrete domain $(\Omega)$ is surrounded by the surface of the concrete $\left(\Gamma_{n}\right)$ and the surface of the steels matrices $\left(\Gamma_{m}\right)$. The steel matrices are divided into corroded and non-corroded parts.

In the previous work [2], the Laplace Equation in Equation (1) has been used to describe the potential in the $\Omega$. The related boundary conditions (BCs) are given in Equation (2) through Equation (3).

$$
\begin{aligned}
& \nabla^{2} \phi=0 \quad \text { in } \Omega \\
& i\left(\equiv \kappa \frac{\partial \phi}{\partial n}\right)=0 \quad \text { on } \Gamma_{n} \\
& -\phi_{m}=\left\{\begin{array}{lc}
f_{m_{1}}(i) & \text { on } \Gamma_{\mathrm{m}_{1}} \\
f_{m_{2}}(i) & \text { on } \Gamma_{\mathrm{m}_{2}}
\end{array}\right.
\end{aligned}
$$

If all of the BCs are prescribed, the direct BEM can be used to solve the Laplace's equation in Equation (1). Hence, the potential $(\phi)$ and current density ( $i$ ) on the whole concrete domain can be determined.

In actual cases, however, the BCs in Equation (3) are unknown. Therefore, an inverse analysis is necessary in order to detect the corrosion profile that represents the number, location and shapes of the plural corrosion parts on the steel matrices in the concrete structure. 
The steel matrices were discretized into k segments

\begin{tabular}{|c|c|c|c|c|c|}
\hline$\ldots$ & $\ldots$ & & & $\cdots$ & $\mathrm{a}_{\mathrm{k}}$ \\
\hline$\vdots$ & & & & & $\vdots$ \\
\hline & & & & & \\
\hline$\vdots$ & $\ldots$ & & & & $\vdots$ \\
\hline $\mathrm{a}_{1}$ & $\mathrm{a}_{2}$ & $\ldots$ & & & $\cdots$ \\
\hline
\end{tabular}

Representation of a corrosion profile, $\mathrm{C}_{\mathrm{k}}$

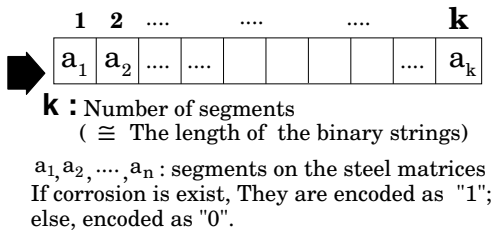

Figure 2: Representation of the corrosion profile.

To simplify the inverse problem, the polarization curves of corroded and noncorroded parts of the steel matrices are prescribed. The inverse analysis is carried out by minimizing a cost-function, $\varepsilon\left(C_{k}\right)$, in Equation (4).

$$
\varepsilon\left(C_{k}\right)=\sum_{l=1}^{N}\left(\frac{\phi\left(x_{l}, y_{l} ; C_{k}\right)-\bar{\phi}\left(x_{l}, y_{l}\right)}{\bar{\phi}_{\max }}\right)^{2}
$$

where $C_{k}$ is the corrosion profile on the steel matrices which is represented by a binary string. The $x_{l}, y_{l}$ are the location of potential measurements on the structure in $\mathrm{x}$ and y directions. The $N$ is the number of measurements, the $\phi$ and $\bar{\phi}$ are the calculated and measured potential values, respectively. The calculated values of potential were obtained by solving the Laplace's equation in Equation (1) using BEM.

\section{The multi-step genetic algorithm}

To define the binary string of the $C_{k}$, the steel matrices is discretized into a suitable number of segments using a certain resolution as given in Figure 2. The number of segment indicates the length of the binary string. The corroded and non-corroded segments are encoded by a binary bit " 1 " and " 0 ", respectively.

When a large area of the steel matrices is discretized using a required resolution, however, a very long binary string is necessary to represent all of the segments. Therefore, a long calculation time is required in carrying out the standard GA.

A tree structure, shown in Figure 3, is employed in the multi-step GA to eliminate the above difficulty. In this method, the examination is performed separately for the root and each branch. Hence, for each step of examination, the standard GA can be applied locally and performed efficiently.

In the first step, to define the binary strings of the $C_{k}$, a low resolution (coarse segment) is used to discretize the entire steel matrices into a suitable number of segments.

The standard GA is carried out by employing some potential data which are spread out uniformly on the surface of the structure. The $\varepsilon\left(C_{k}\right)$ in Equation (4) is used to measure the fitness of each binary string as a candidate of the true $C_{k}$. Two conditions were used to terminate the iteration process: (1) the $\varepsilon\left(C_{k}\right)$, is smaller 


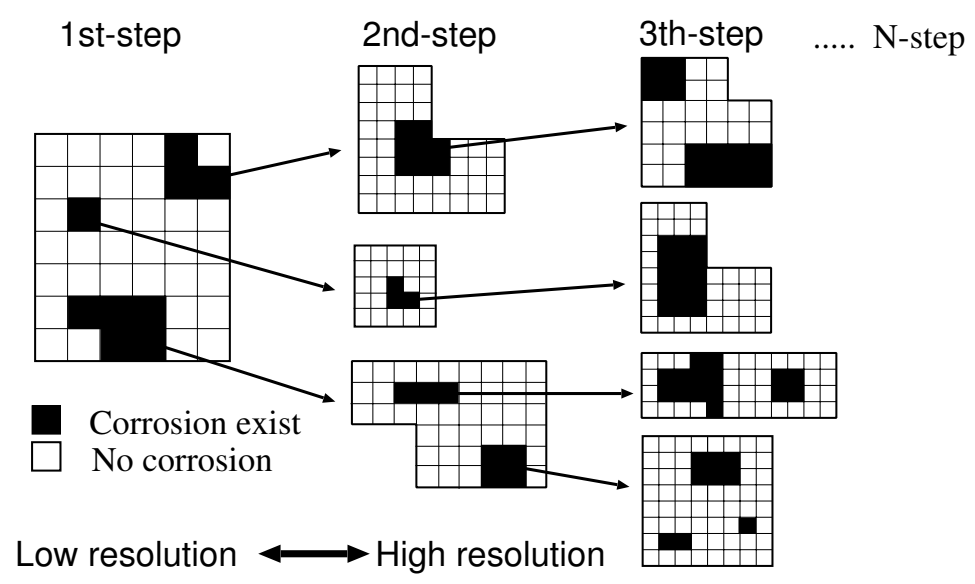

Figure 3: Concept of the multi-step GA.

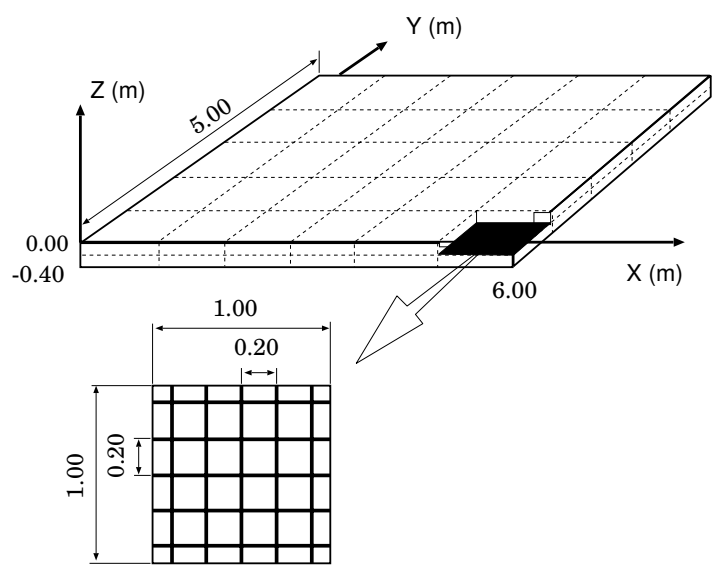

Figure 4: Model of the reinforced concrete specimen and detail of the steel matrices.

than $\varepsilon_{o}$, or (2) the number of generations in the GA is reached. From this step, the corrosion is localized and one or more corroded parts are obtained.

In the second and next steps, the examination is carried out separately for each corroded part. The local steel matrices is remeshed into a certain segment using a higher resolution. The procedures of the GA is the same as the previous step but they may used a smaller size of the segments, a different length of the binary string and a different number of populations and generations. 


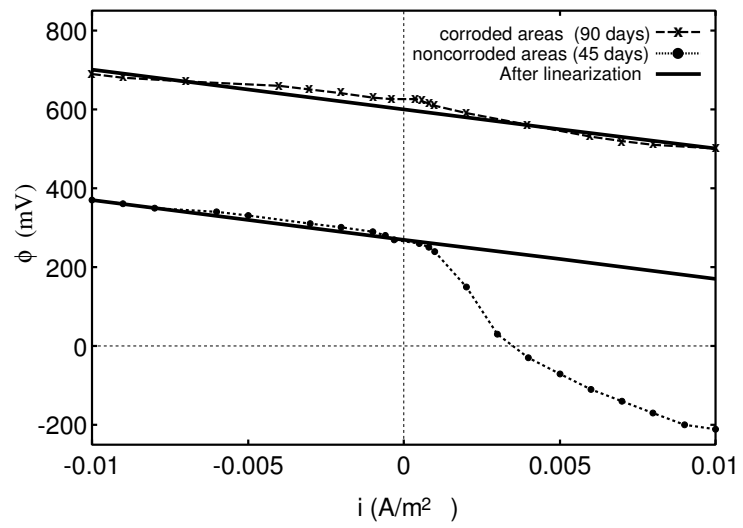

Figure 5: Two linear and parallel polarization curves for corroded and noncorroded parts of the steel matrices. These curves were approximated from the literature [5].

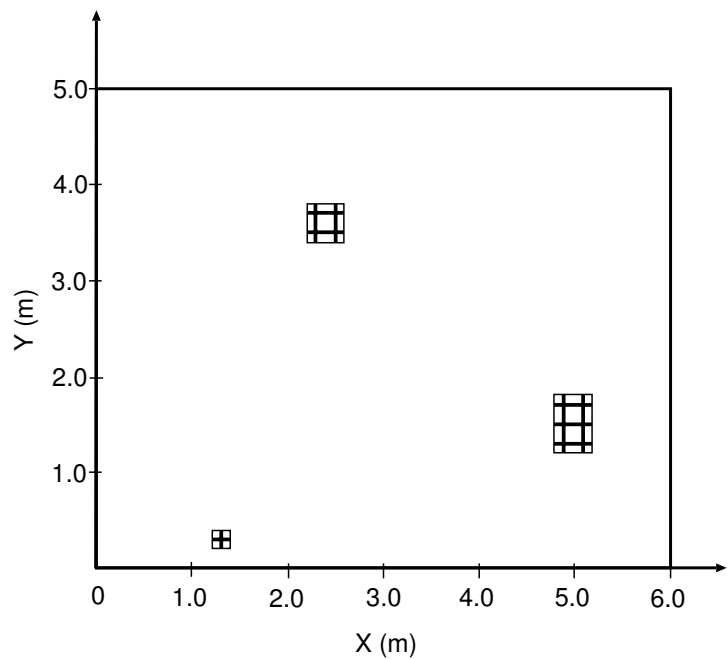

Figure 6: The profile of corrosion area on the steel matrices that were used in direct BEM.

The final step is carried out when the area of examination become suitable to be discretized using the high resolution. Once this condition is achieved, then the standard GA can be performed to accurately detect the corrosion profile on the steel matrices. 


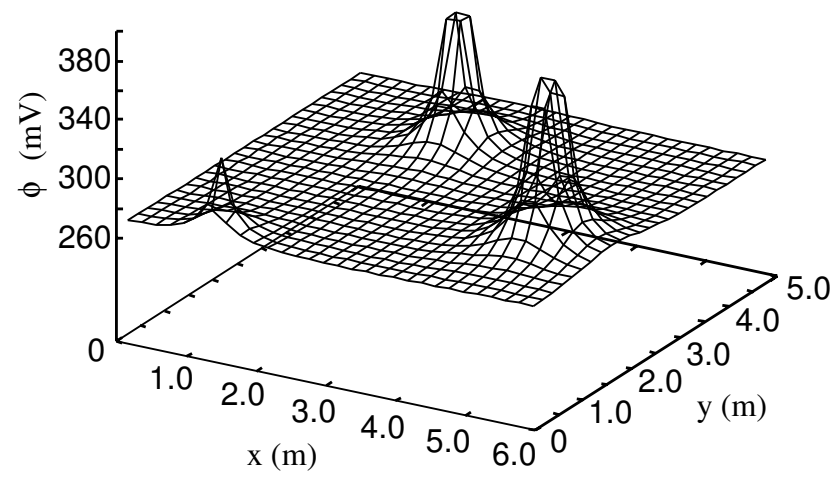

Figure 7: Potential distribution on the surface of the concrete specimen (result of the direct BEM calculation).

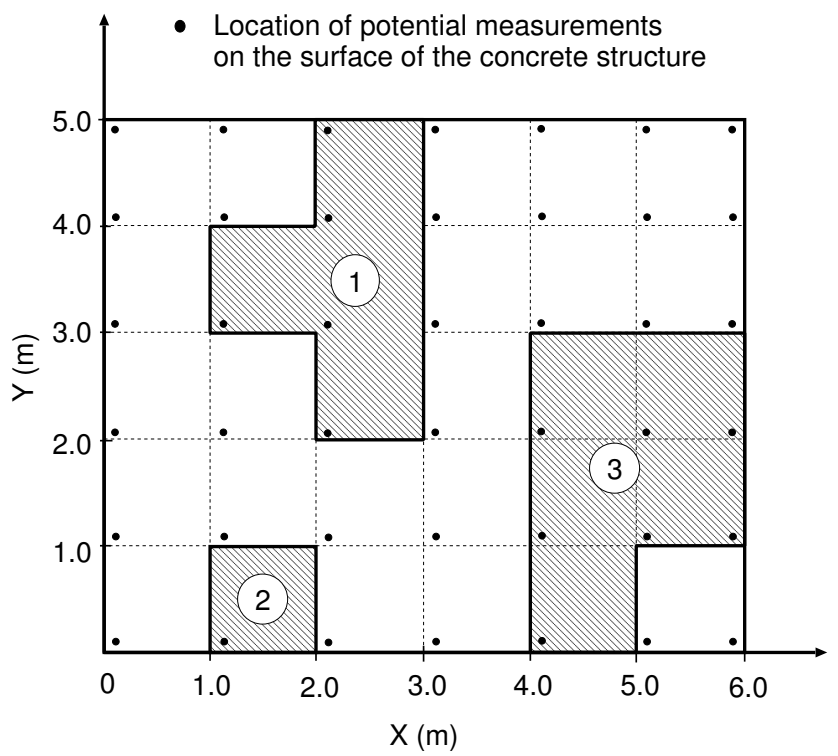

Figure 8: Result of the first-step of the multi-step genetic algorithm and the locations of potential measurements on the surface of the concrete structure.

\section{Numerical simulation and discussion}

An example using simulation data was used to verify the effectiveness of the proposed multi-step genetic algorithm. A preliminary numerical simulation was carried out to obtain the potential values on the surface of the concrete structure. 


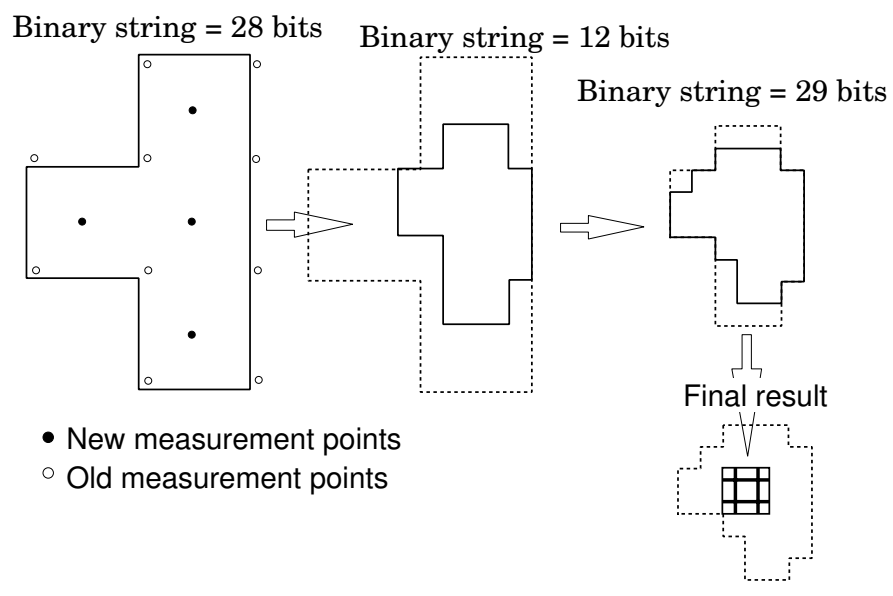

Figure 9: The multi-step genetic algorithm for detecting the first corroded part.

By giving all of the boundary conditions in Equation (2) and Equation (3), the Laplace's equation in Equation (1) was solved directly using BEM.

For carrying out the multi-step genetic algorithm, a small number of potentials were chosen from the obtained potential values. These potential values were rounded off into three digits in order to simulate modeling and measurement errors and used as the measured potential data.

\subsection{Model of the reinforced concrete structure}

We considered a model of reinforced concrete specimen shown in Figure 4, where the electrical conductivity $\left(\kappa\right.$ ) of the concrete was $7.10^{-3} 1 / \Omega . \mathrm{m}$, the steel matrices were cast in the concrete in the depth of $0.07 \mathrm{~m}$, the diameter of the steels were $0.01 \mathrm{~m}$, and the distance between two steels in the concrete specimen was $0.2 \mathrm{~m}$ in both of $\mathrm{x}$ and $\mathrm{y}$ directions.

The steel matrices were divided into corroded and non-corroded parts. Two polarization curves of the steel in concrete which were immersed in a $10 \%$ $\mathrm{NaCl}$ solution for 90 days $\left(E_{\text {corr }}=-0.635\right.$ Volt vs. SCE.) and 45 days $\left(E_{\text {corr }}=-0.270\right.$ Volt vs. SCE.), that were reported by Wheat et al. [5], were employed to represent the polarization curves of the corroded and non-corroded parts of the steel matrices, respectively.

To reduce the computational time, we linearized the polarization curves as shown by solid-line in Figure 5. The formula of the linear curves for the corroded and non-corroded parts were $\phi_{a}=-10 . i+0.600$ Volt and $\phi_{c}=-10 . i+$ 0.270 Volt, where $i$ was the current density $\mathrm{A} / \mathrm{m}^{2}$. It noted that when the original non-linear curve is used, the proposed method also can be carried out normally, but the calculation time for each iteration of BEM calculation became increase. 


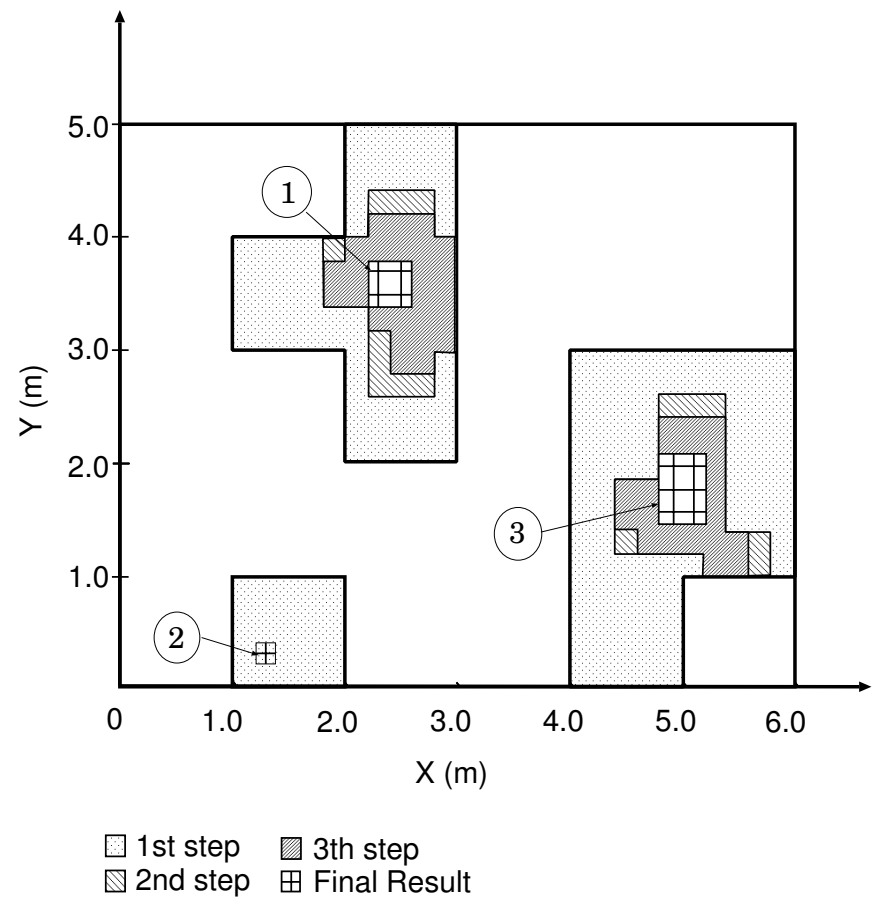

Figure 10: Result of the multi-step genetic algorithm.

\subsection{Direct boundary element calculation}

Three corroded parts were assumed on the steel matrices in the concrete specimen. Their locations and shapes were given in Figure 6.

In the BEM calculation, the surface of the steel matrices was discretized into 1500 constant elements, while the surface of the concrete, except for the top surface, were discretized into 970 constant elements. The type of the elements for the concrete and steel were the rectangular element (size $0.2 \times 0.2 \mathrm{~m}$ ) and the pipe element (length $0.2 \mathrm{~m}$ and diameter $0.01 \mathrm{~m}$ ), respectively. The potential value at any point on the top surface of the concrete specimen were calculated by using the method of images [7].

Figure 7 shows the potential distribution on the surface of the specimen as a result of the direct BEM calculation. In order to simulate modeling and measurement errors in carrying out the multi-step genetic algorithm, however, only about 50 values of the potentials were chosen and rounded off into three digits. These potential values were used as the measurement data of potential on the surface of the concrete specimen. 


\subsection{Result of the multi-step genetic algorithm}

In order to carry out the multi-step genetic algorithm for solving the inverse problem using the simulation data, firstly, let us assumed that the number, locations and shapes of corrosion on the steel matrices were unknown and only a small number of potential data on the surface of the concrete specimen were available. The electrical conductivity $(\kappa)$ of the concrete and the polarization curves of corroded and non-corroded steels were assumed to be the same as in the previous sections.

A multi-step genetic algorithms was employed to obtain the corrosion profile $\left(C_{k}\right)$ on the steel matrices in the concrete structure. The $C_{k}$ was encoded by a binary string. For each step of examination, we used the genetic algorithm driver which was developed by D. L. Carroll [6].

In the first step of examination, to define the length of the binary string of the $C_{k}$, the steel matrices were discretized using a low resolution (coarse mesh) into 30 segments. Each segment consisted of 50 steel-elements.

Forty-two simulation data of potential, which uniformly spread out in the entire surface of the concrete structure, were employed as the measurement values of potential on the surface of the concrete structure. The distance between two locations of measurement was one meter.

The genetic algorithm was carried out by setting the number of populations of 50 and the number of generations of 100 . In addition, the tolerance $\left(\varepsilon_{o}\right)$ was $10^{-5}$. The result of the first-step of the multi-step genetic algorithm was given in Figure 8 . It can be seen that the corrosion was localized into three parts on the steel matrices.

In the second and next steps, the examination was localized for each corroded parts. The procedures of the genetic algorithm were the same as in the first step, but it may uses a smaller size of segment, different length of the binary strings, some new measurement data and/or different number of populations and generations. Figure 9 shows result of the multi-step genetic algorithm for detecting the the first corroded part. In examining the corrosion of this part, three new measurement data were added. The accurate result of the corrosion profile was obtained after performing the forth-step of the standard genetic algorithm.

Similar procedures were also carried out for detecting the second and third corroded parts that were obtained in the previous step. The result of the multisteps genetic algorithm for identifying the corrosion on the steel matrices was summarized in Figure 10. It can be seen that the second and third corrosion parts were accurately detected after carrying out two and four steps of the standard genetic algorithm, respectively.

For the given example, the complete examination process consisted of 8 steps of the genetic algorithm. The calculation time for the complete problem to convergence was about 7 hours (using VT-Alpha 6-667, 667 MHz, OS:Tru64 Unix, memory 2G). However, Since each corroded part is examined separately, then we can employ parallel computation to reduce the calculation time for the complete problem to convergence. 


\section{Conclusions}

A multi-step GA was developed to eliminate the difficulties of the inverse method for detecting the corrosion profile from a small number of potential data. The corrosion profile represents the number, locations and shapes of corrosion on the steel matrices. It is encoded by a binary string which is defined by discretizing the steel matrices into a number of segments. A tree structure was employed and the examination was localized for the root and the branches. Therefore, the standard GA can be applied locally and performed efficiently. An example using simulation data was used to demonstrate the effectiveness of the proposed method.

\section{References}

[1] J. P. Broomfield: "Corrosion of Steel in Concrete", London: E \& FN Spon (1998).

[2] M. Ridha, K. Amaya \& S. Aoki, Proc.12th Compt. Mech. Conf., JSME, 461462 (1999).

[3] S. Aoki, K. Amaya \& M. Miyasaka: Boundary Element Analysis on Corrosion Problems, Shokabo (1998).

[4] D. E. Goldberg, "Genetic Algorithms in Search, Optimization and Machine Learning”, Addison- Wesley (1989).

[5] H. G. Wheat, Z. Eliezer, Corrosion, 41, 11, 640-645 (1985).

[6] D. L. Carroll, "FORTRAN Genetic Algorithm Driver", http://www.staff.uiuc.edu/ carroll/ga.html, (1998).

[7] C. A. Brebia, The Boundary Element Technique in Engineering, (London: Newnes-Butterworths, 1980), p.102. 